\title{
Assessment the Optimal Effect of Time of Repetition: Extrinsic Pulse Parameter on Gd-DTPA Enhanced, Spin-Echo T1-Weighted MR Images under Low Magnetic Field Strength
}

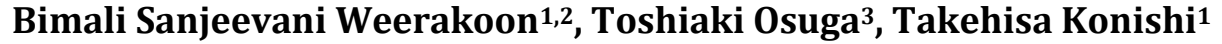 \\ ${ }^{1}$ Graduate School of Advanced Integration Science, Chiba University, Chiba, Japan \\ ${ }^{2}$ Department of Radiography/Radiotherapy, Faculty of Allied Health Sciences, University of Peradeniya, \\ Peradeniya, Sri Lanka \\ ${ }^{3}$ Center for Frontier Medical Engineering, Chiba University, Chiba, Japan \\ Email: bsw888@gmail.com
}

Received 20 July 2016; accepted 28 August 2016; published 31 August 2016

Copyright (C) 2016 by authors and Scientific Research Publishing Inc.

This work is licensed under the Creative Commons Attribution International License (CC BY). http://creativecommons.org/licenses/by/4.0/

c) (i) Open Access

\section{Abstract}

The contrast agent concentration, the time of repetition (TR) and magnetic field strength are significant parameters that influence for the accurate signal intensity (SI) in quantitative Magnetic Resonance Imaging (MRI). Therefore, this study was conducted to investigate and refine the dependence and the optimal effect of Time of Repetition (TR) on the relationship between signal intensity and Gd-DTPA (Gadolinium-diethylene-triaminepenta-acetic acid) concentration, after applying two-dimensional (2D) Spin Echo (SE) pulse sequence under low-field MRI. In addition to that, the optimal concentration of Gd-DTPA at given sequence parameters at low-field MRI was also evaluated. A water-filled phantom was constructed for a range of Gd-DTPA concentrations ( 0 - 6 $\mathrm{mmol} / \mathrm{L}$ ) and the mean signal intensities (SIs) were assessed in the defined region of interest on T1-weighted images with different TR values $(40-2000 \mathrm{~ms})$. The generated signal-concentration curves for Gd-DTPA revealed that increasing TR was associated with the increase of the overall SIs and the maximum relationship between SI to concentration. Moreover, the required Gd-DTPA concentration to produce the maximum SI was associated to decrease with the increase of TR. In addition to this, the application of beyond $100 \mathrm{~ms}$ TR values in this study with relatively higher concentrations (beyond $1-2 \mathrm{mmol} / \mathrm{L}$ ) has resulted predominantly non-linear patterns in the signal-concentration curves and it appears the saturation or decay of the SIs due to T2 effect. From these results, it can be suggested that the selection of relatively lower Gd-DTPA concentration $(<1$

How to cite this paper: Weerakoon, B.S., Osuga, T. and Konishi, T. (2016) Assessment the Optimal Effect of Time of Repetition: Extrinsic Pulse Parameter on Gd-DTPA Enhanced, Spin-Echo T1-Weighted MR Images under Low Magnetic Field Strength. International Journal of Medical Physics, Clinical Engineering and Radiation Oncology, 5, 196-203. 
mmol/L) with less than $800 \mathrm{~ms}(<800 \mathrm{~ms})$ TR values can produce a better linear relationship between the concertation and SIs in T1-weighted SE low field contrast-enhanced MRI. Furthermore, this study also outlined the significance and necessity of the optimization of TR in SE sequence in low field MRI prior to a particular examination.

\title{
Keywords
}

\author{
Gd-DTPA Concentration, Spin Echo Pulse Sequence, Signal Intensity, Time of Repetition, \\ T1-Weighted Images, Low Field MRI
}

\section{Introduction}

After introducing Magnetic Resonance Imaging (MRI) contrast agents (CAs) into the clinical setting, it has become a powerful medical imaging tool with a wide array of applications [1]. Gadolinium $\left(\mathrm{Gd}^{3+}\right)$ based paramagnetic agents are the most commonly utilized CAs in the clinical setting as they have a range of applicability [2] [3]. Due to the low intrinsic sensitivity of MRI, the higher local concentration of the CA at the target site may be necessary to produce higher image contrast [2]. The application of the higher level of concentration is able to increase the signal to noise ratio on perfusion images [4]. Nevertheless, the contrast enhancement of GdDTPA (Gadolinium-diethylene-triaminepenta-acetic acid) does not linearly correlate with higher concentrations [5], [6]. At lower concentrations $T 1$ shortening effect becomes more dominant, whereas at higher concentrations of Gd-DTPA, T2 shortening effect becomes more dominant, which causes deterioration in signal intensity [6], [7]. Furthermore, the intrinsic and extrinsic factors such as proton density, spin relaxation time [5], image acquisition parameters [4], [8], magnetic susceptibility effect [9], magnetic field strengths [1] and type of pulse sequence [6], [8] also play significant roles in the association of signal enhancement and quality of the acquired information.

Although most of the current diagnostic imaging and research is actively engaged in high-field MRI, more inexpensive way to perform experiments is the utilization of low-field MRI [10]. In addition to this, low-field open MRI provides more convenient and more comfortable examination procedures for a patient [11], specifically, one with heavy-weight [12] and claustrophobia. Moreover, low-field MRI can minimize the chemical shift and orthopaedic hardware artifacts and facilitate high magnetic field homogeneity better than the high field MRI [12]. Therefore, a comprehensive determination of the effects of imaging parameters and contrast concentration in low-field MRI is vital to produce optimum signal intensity for diagnostic and economic implications, for new clinical applications and screening procedures [10].

In the literature, the effect of time of repetition (TR/time between two successive excitation RF pulses or time between consecutive $90^{\circ} \mathrm{RF}$ pulses [13]) [4], time of echo (TE) [14], time of inversion (T1) [8] and magnetic field strength [1] on the signal intensities (SIs) with different types of contrast agents and concentrations using various pulse sequences have been discussed frequently. Thus far, the optimization of time of repetition (TR) on a range of Gd-DTPA concentrations in low field MRI using spin-echo (SE) sequence has not received sufficient attention. $T R$ is an extrinsic pulse parameter which can be controlled by the operator to produce optimum $T 1, T 2$ or PD images. In addition to this, TR is one of the parameters which controls the overall scan time [15]. At the same time, $S E$ sequence is the most readily available pulse sequence in the clinical settings with higher signal to noise ratio, a wide range of contrast behaviour ( $T 1$ or $T 2$ or PD-weighted), and insensitivity to signal loss caused by external field in homogeneities [16]-[19].

Therefore, this paper seeks to investigate the dependence and the optimal effect of TR into the relationship between the signal intensity and Gd-DTPA concentration, after applying two-dimensional (2D) Spin Echo (SE) pulse sequence under low-field MRI. In addition to that, the optimal concentration of Gd-DTPA at given sequence parameters under low-field MRI was also evaluated and it may provide the guidance on contrast-enhanced imaging procedures.

\section{Theoretical Background}

The longitudinal relaxation time ( $T 1$ ) maps can be constructed by applying a series of $T 1$-weighted images at different $T R$ values from spin-echo sequence on the solution to the Bloch equation as below: 


$$
S=S_{0}\left(1-\exp ^{-\frac{T R}{T 1}}\right) \exp ^{-\frac{T E}{T 2}}
$$

where $S, T R$, and $T 1$ are the signal intensity, time of repetition and longitudinal relaxation time, respectively. $S_{0}$ is the signal intensity of the medium after complete $T 1$ relaxation at $T E=0$, which controls by the proton density and instrument factors. TE and T2 are the time of echo, and transverse relaxation time [17], [20]-[22]. Because T1-weighted images were obtained withconstant TE value andwith the condition of $T E<<T 2$ [17], the SI of Equation (1) could be simplified as below [23]:

$$
S \alpha\left(1-\exp ^{-\frac{T R}{T 1}}\right)
$$

The slope of the regression curve between the longitudinal relaxation rate $(1 / T 1)$ and contrast concentrations (C) gives the $T 1$ relaxivity constant of the contrast agent $(r 1)$ as expressed below [24]: where the $1 / T 10$ is the relaxation rate of the solvent without application of contrast solution.

$$
\frac{1}{T 1}-\frac{1}{T 10}=r 1 . C
$$

\section{Materials and Methods}

\subsection{Phantom Construction}

Two phantoms constructed with nine vials in each (plastic $3 \mathrm{ml}$ vials which have the approximate inner diameter of $10 \mathrm{~mm}$ ) were applied to get the measurements (Figure 1). The vials of the phantom filled only with distilled water were used to measure the field in homogeneity. Another Gd-DTPA mixed, a distilled water-based (Magnevist, Schering, Germany) multiple vial phantom was constructed with a known range of Gd-DTPA concentrations $(0,0.1,0.25,0.5,0.75,1,2,4,6 \mathrm{mmol} / \mathrm{L})$ (greater than the physiologic range $(0-2 \mathrm{mmol} / \mathrm{L})$ [25]) to investigate the optimum effect of $T R$ on signal enhancement. The surrounding area of the vials was filled with distilled water.

\subsection{Image Acquisition}

All imaging was performed using a Hitachi AIRIS Vento (Hitachi Medical Corporation, Tokyo, Japan) whole body open clinical MR scanner operated at $0.3 \mathrm{~T}$ with the birdcage head coil. The vials of the phantom were placed vertically in the is o-centre of the scanner with their longitudinal axes perpendicular to the imaging plane. The T1-weighted images were acquired from the two-dimensional (2D) SE pulse sequence with variable times of $T R(40,60,80,100,200,300,400,600,800,1000,2000 \mathrm{~ms})$. Minimum possible TE value of $13.1 \mathrm{~ms}$ was selected to minimize the $T 2$ effect. Other selected imaging parameters were: flip angle $=90^{\circ}$, acquisition matrix size $=256 \times 192$, field of view $(F O V)=180 \mathrm{~mm} \times 180 \mathrm{~mm}$, number of signal averages $(\mathrm{NSA})=4$. According to the structure of the vials, 2 - 4 trans-axial images (Figure 1) were acquired in each TR value. Slice thickness and

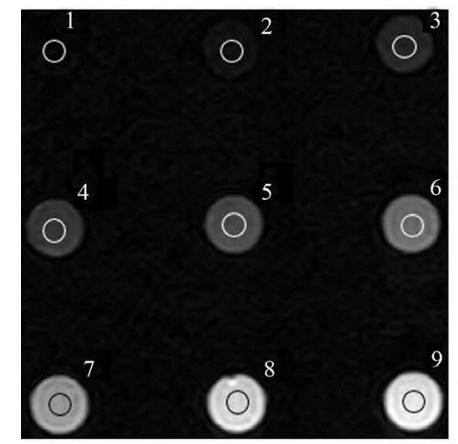

(a) $\mathrm{TR}=60 \mathrm{~ms}$

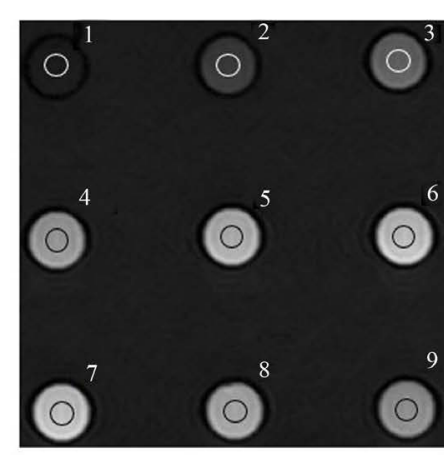

(b) $\mathrm{TR}=400 \mathrm{~ms}$

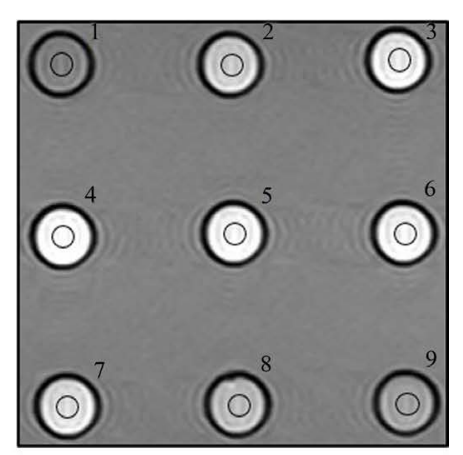

(c) $\mathrm{TR}=2000 \mathrm{~ms}$

Figure 1. The trans-axial images of the Gd-DTPA mixed phantom taken at $T R=60,400$ and $2000 \mathrm{~ms}$. The arrangement of $1-9$ vials of each image illustrate the Gd-DTPA concentrations of $0,0.1,0.25,0.5,0.75,1,2,4$ and $6 \mathrm{mmol} / \mathrm{L}$, respectively. Defined ROI is demonstrated by a black or white circular outline inside the vials. 
inter-slice gap were selected as $5 \mathrm{~mm}$ and $7 \mathrm{~mm}$, respectively. The temperature was maintained at approximately $25^{\circ} \mathrm{C}$ within the gantry and the phantom and monitored every 30 minutes during the scan period. Two image acquisitions were performed with the same parameters and the results were averaged.

\subsection{Image Analysis}

The mean signal intensities of each vial were measured using Image $J$ (National Institutes of Health, USA) software by placing a circular region of interest (ROI) including fifteen innermost pixels of the vials to avoid the partial volume effects. The background noise and field in homogeneity were corrected by considering the mean signal intensity values of the phantom which only consisted with vials of distilled water. After three repetitive measurements averaged signal intensities to concentration curves were generated at different $T R$ values to identify the optimum effect.

\section{Results}

\subsection{Signal-Concentration Curves at Range of $T R$}

Sample MR images correspond to $T R$ values of 40,400, and 2000 ms were demonstrated in Figure 1. The mean signal intensities as a function of Gd-DTPA concentrations with respect to the different $T R$ values from $40 \mathrm{~ms}$ $2000 \mathrm{~ms}$ have been presented in Figure 2. According to the resulted curves, the maximum signal intensities were reported at different concentrations in different $T R$ values. However, the SI enhancement was approximately linearly proportional to the Gd-DTPA concentration less than $1 \mathrm{mmol} / \mathrm{L}$ in most of the selected TR values. Moreover, overall SIs were increased with increasing $T R$.

\subsection{The Obtained Maximum Signal Intensities at Different TR Values}

Figure 3 demonstrates the relationship between the obtained maximum SIs with different $T R$ values. This graph indicates that the maximum SI gradually increases when the TR is increased up to $1000 \mathrm{~ms}$ in this study, but after $1000 \mathrm{~ms}$ it appears to be saturated.

\subsection{The Maximum Signal Intensities and the Required Gd-DTPA Concentration}

The relationship between the different Gd-DTPA concentrations which exhibit the maximum SIs with a range of TR has been presented in Figure 4. When TR is increased, the required Gd-DTPA concentration which leads to the maximum SI is decreased gradually.

\subsection{The Ratio between the Maximum Signal Intensity and the Required Gd-DTPA Concentration}

The ratios between the maximum SIs at each $T R$ value and the required Gd-DTPA concentrations which produced

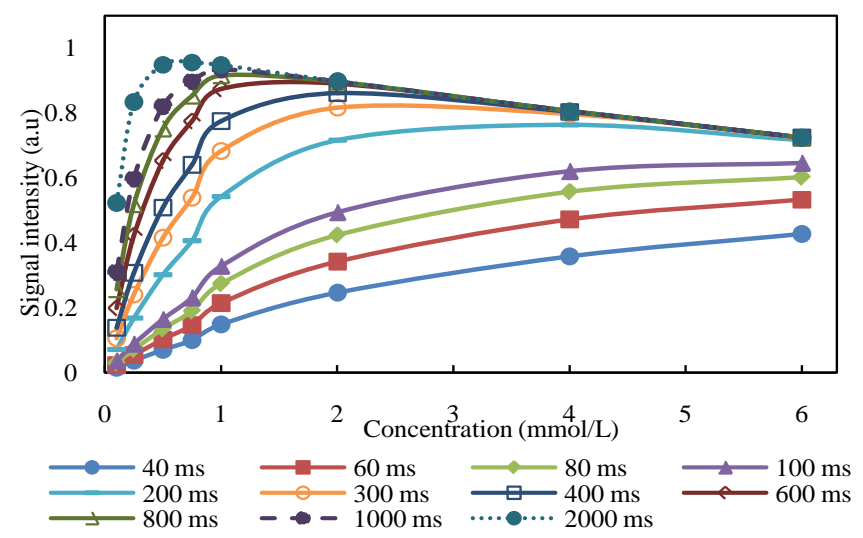

Figure 2. The obtained mean Sis as a function of the contrast agent concentrations, at different $T R$ values which range from $40 \mathrm{~ms}$ 2000 ms. 


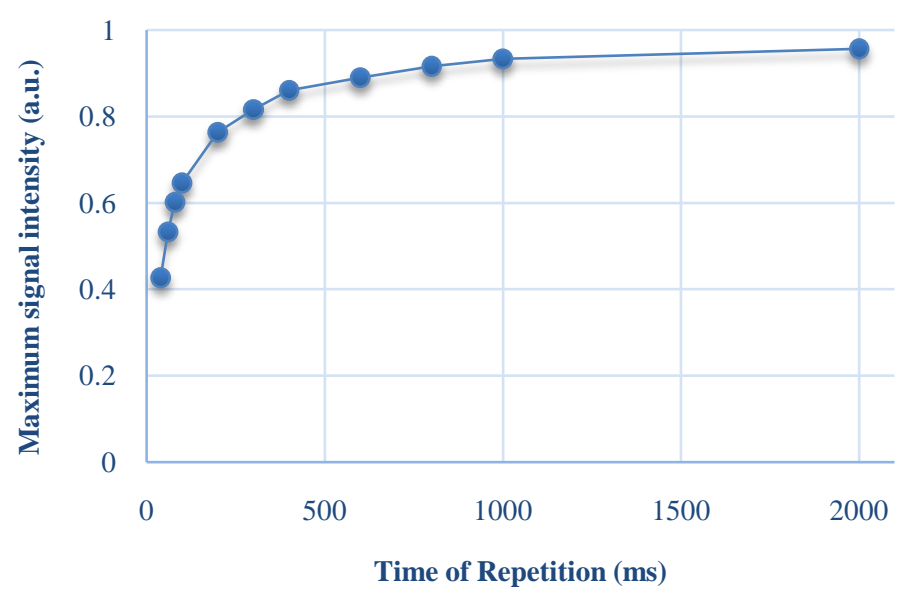

Figure 3. TR versus obtained maximum SIs. The maximum SI is increased gradually up to $T R=1000 \mathrm{~ms}$, then appears to be saturated.

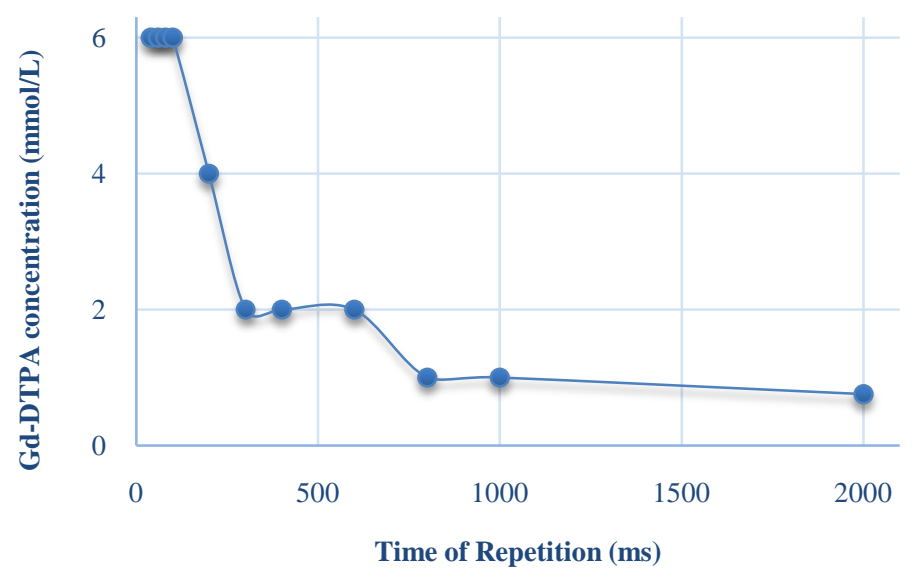

Figure 4. The Gd-DTPA concentration which required for producing the maximum SIs at each $T R$ value. This concentration decreases when TR increases.

these maximum SIs are plotted in Figure 5. Based on this figure, the maximum SI, with lowest possible contrast concentration was achieved at $T R$ value of $2000 \mathrm{~ms}$ in this study.

\section{Discussion}

As mentioned before the concentration of Gd-DTPA does not linearly correlate with the Sis [5], [6]. Furthermore, intrinsic and extrinsic parameters [4] also play an important role in the association of signal enhancement. The applied pulse sequence [6] and the magnetic field strength [1] are extrinsic parameters which have a major influence on signal enhancement. Therefore, in this study, the dependencies of Gd-DTPA signal intensities on the $T R$ values at various concentration levels were quantified in low-field MRI with SE [26] pulse sequence using the serial dilution method [4], [5].

Indeed the $T R$ will directly affect the level of $T 1$ weighting [17]. Optimal $T 1$-contrast can be achieved using a relatively short $T R(T R \approx T 1)$ whereas the contrast is more weighted towards PD when $T R>T 1$. However, even with long $T R$ values, the resulted images can be expressed $T 1$-weighting factor into some extent on mediums with long T1-relaxation time [15], [17]. According to the study results (Figure 2), when the selected TR values were progressively lengthened from 40 to $2000 \mathrm{~ms}$, the overall SIs tended to increase. Furthermore, the required concentration of the contrast agent, which leads to the maximum SI, also depended on $T R$ and this value decreased with the increase of $T R$ (Figure 4). When applying the lower $T R$ values such as $40 \mathrm{~ms}$, a positive correlation of SI was obtained at concentration levels up to $6 \mathrm{mmol} / \mathrm{L}$ but when $T R$ value was higher (at 


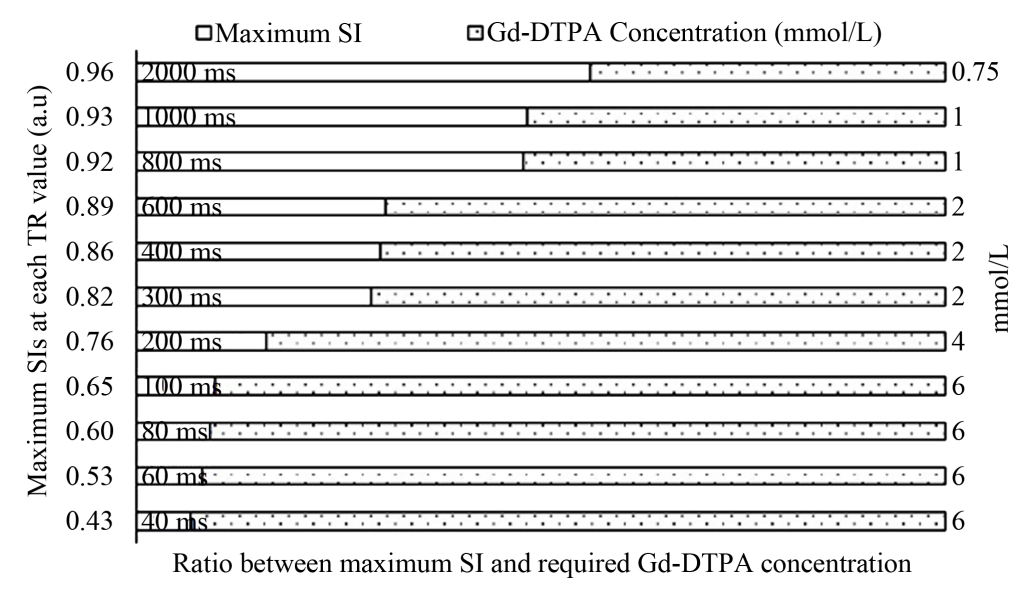

Figure 5. The ratios between the reported maximum SIs at each $T R$ values and the required Gd-DTPA concentrations which produced these maximum SIs.

$T R=2000$ ), the positive correlation was reported only up to $0.5 \mathrm{mmol} / \mathrm{L}$ (Figure 2). However, according to the obtained results, the SI enhancement was approximately linearly proportional to the Gd-DTPA concentration less than $1 \mathrm{mmol} / \mathrm{L}$ in most of the selected $T R$ values.

The $T 1$ relaxation time of most samples increases as the magnetic field increases due to the increase in water tumbling, which causes a mismatch between hydrogen and lattice [27]. At low magnetic fields, on the other hand, $T 1$ is short, which requires the use of short $T R$ to preserve the $T 1$ image contrast. The presence of Gdbased contrast media further reduces the $T 1$ relaxation time making use of short $T R$ to maintain the $T 1$ contrast. As illustrated in Figure 1, the contrast between different Gd-DTPA samples was more pronounced on the image acquired with a shorter repetition time ( $T R=40 \mathrm{~ms}$ ), because of the stronger $T 1$ weighting. Furthermore, shorter $T R$ will move the turn over-seen to higher Gd-DTPA concentration [5].

As expected from Equation (3), Figure 2 confirmed the behaviour of the Gd-DTPA which demonstrated the dominant $T 1$-shortening effect at lower concentrations by increasing the SIs, whereas the dominant $T 2$-shortening effect at higher concentrations by the appearance of saturation or decay of SIs. This behaviour was in good agreement with the previously reported studies [4]-[6] with Gd-DTPA in different mediums. However, this reflects the idea that although the image is highly $T 1$-weighted, at higher concentrations the signal response became non-linear due to the $T 2$ (or $T 2 *$ ) effect [15], [28]. This process is more prominently visible with the application of longer $T R$ values, as explained by Equation (3). At a high concentration of the Gd-DTPA with longer $T R$, signal plateaus are caused due to complete $T 1$ relaxation processes (or almost completion) between pulses, resulting PD-weighted images instead of $T 1$ weighted [5]. A previous study done with SE T1-weighted imaging at $0.3 \mathrm{~T}$ demonstrated a similar pattern: which the SIs were linearly proportional to Gd-DTPA concentrations at lower levels whereas it became non-linear at higher concentrations. In addition to this, maximum SI was detected at $5.95 \mathrm{mmol} / \mathrm{L}$ with the selection of $T R / T E=411 \mathrm{~ms} / 15 \mathrm{~ms}$ in the above study [6]. But the reported maximum SI at TR/TE $=400 \mathrm{~ms} / 13.1 \mathrm{~ms}$ in the current study was $2 \mathrm{mmol} / \mathrm{L}$. Although an exact aetiology can't be definitively determined, this disparity may arise from the effect of temperature or other parameter difference. Another in-vitro study conducted with $S E$ under $0.35 \mathrm{~T}$ magnetic strength (Nuclear Magnetic Resonance imaging unit) demonstrated that the Sis were enhanced from different degrees, in the range of $0-5 \mathrm{mmol} / \mathrm{L}$ Gd-DTPA concentrations with TR/TE ratio of 500/28. The highest possible signal intensity in that study was observed at $1.5 \mathrm{mmol} / \mathrm{L}$ [29]. These results confirm the high sensitivity of Gd-DTPA SIs on the extrinsic pulse parameters.

The ratio between the maximum SIs at each $T R$ value and the required concentrations to produce these maximum SIs (Figure 5) revealed that the maximum signal enhancement can be obtained at lower concentrations (0.75 mmol/L) when higher $T R$ values (2000 ms) are applied. Conversely, it is important to mention that increasing $T R$ causes to increase the scan time since the scan time in a $S E$ sequence is directly proportional to $T R$ [15]. Moreover, a higher $T R$ value weakens the $T 1$-weighted contrast. Therefore, when applying conventional $S E$ sequence, the maximum $T R$ is a limited factor.

As noted above, different papers have reported different values for the maximum linear relationship between 
SI and the concentration of contrast agents in T1-weighted imaging. However, these results with the current experiment clearly indicate that $T R$ is one of the several significant extrinsic image parameters affecting the strength of the SI depending on the contrast agent concentration even at low-field MRI. Therefore, the optimum balance between the TR, SI, and contrast concentration is necessary to produce ideal MR images. As demonstrated in this study, it is suggested to perform a phantom experiment to calibrate and optimize the pulse parameters prior to quantitative imaging as because there is no standard theoretical way to choose the optimal pulse parameters under different settings which require for precise estimation and diagnosis [25].

\section{Conclusions}

An optimal $T R$ value is essential in any magnetic field for deriving an accurate signal to concentration curves in quantitative MRI. Therefore, in this study, the dependence and the optimal effect of $T R$ towards the SI to the concentration of Gd-DTPA were evaluated using 2D-SE pulse sequence in low-field MRI (0.3 T). The results of the present study clearly indicate that $T R$ is one of the major influential factors for generating the SI depending on Gd-DTPA concentrations.

These results revealed that higher $T R$ values produce a non-linear pattern at the higher concentration levels of Gd-DTPA even in low-field MRI under SE sequence. The SI enhancement was approximately linearly proportional to the Gd-DTPA concentration less than $1 \mathrm{mmol} / \mathrm{L}$ in most of the selected $T R$ values. In addition to this, the overall SI tends to increase with the increasing of $T R$ values but it expenses more time with less $T 1$-weighted contrast. Therefore, it can be proposed that the selection of low Gd-DTPA concentration level $(<1 \mathrm{mmol} / \mathrm{L})$ with less than $800 \mathrm{~ms}$ ( $<800 \mathrm{~ms}$ ) TR values can produce a better linear relationship between the concertation and Sis in $S E$ sequence under low field contrast-enhanced MRI. Furthermore, this study also outlined the significance and necessity of the optimization of $T R$ in $S E$ sequence in low field MRI prior to a particular examination.

\section{Acknowledgements}

The authors highly appreciate and are thankful for the support given by the Ministry of Education, Culture, Sports, Science, and Technology (MEXT: Japanese Government MONBUKAGAKUSHO Scholarship program) in Japan.

\section{References}

[1] Rohrer, M., Bauer, H., Mintorovitch, J., Requardt, M. and Weinmann, H.-J. (2005) Comparison of Magnetic Properties of MRI Contrast Media Solutions at Different Magnetic Field Strengths. Investigative Radiology, 40, 715-724. http://dx.doi.org/10.1097/01.rli.0000184756.66360.d3

[2] Riyahi-Alam, S., Haghgoo, S., Gorji, E. and Riyahi-Alam, N. (2015) Size Reproducibility of Gadolinium Oxide Based Nanomagnetic Particles for Cellular Magnetic Resonance Imaging: Effects of Functionalization, Chemisorption and Reaction Conditions. Iranian Journal of Pharmaceutical Research, 14, 3-14.

[3] Kanakia, S., Toussaint, J.D., Chowdhury, S.M., Lalwani, G., Tembulkar, T., Button, T., et al. (2013) Physicochemical Characterization of a Novel Graphene-Based Magnetic Resonance Imaging Contrast Agent. International Journal of Nanomedicine, 8, 2821-2833.

[4] Nazarpoor, M. (2009) The Effect of Repetition Time on the Maximum Linear Relationship between Contrast Agent Concentration and Signal Intensity on T1-Weighted Image Using Inversion Recovery (IR) Sequence. Iranian Journal of Radiology, 6, 247-252.

[5] Shahbazi-Gahrouei, D., Williams, M. and Allen, B.J. (2001) In Vitro Study of Relationship between Signal Intensity and Gadolinium-DTPA Concentration at High Magnetic Field Strength. Australasian Radiology, 45, 298-304. http://dx.doi.org/10.1046/j.1440-1673.2001.00924.x

[6] Nazarpoor, M., Poureisa, M. and Daghighi, M.H. (2012) Comparison of Maximum Signal Intensity of Contrast Agent on t1-Weighted Images Using Spin Echo, Fast Spin Echo and Inversion Recovery Sequences. Iranian Journal of Radiology, 10, 27-32. http://dx.doi.org/10.5812/iranjradiol.5452

[7] Curvo-Semedo, L. and Caseiro-Alves, F. (2011) MR Contrast Agents. In: Gourtsoyiannis, N.C., Ed., Clinical MRI of the Abdomen, Springer-Verlag Berlin Heidelberg, 17-39.

[8] Saharkhiz, H., Gharehaghaji, N., Nazarpoor, M., Mesbahi, A. and Pourissa, M. (2014) The Effect of Inversion Time on the Relationship between Iron Oxide Nanoparticles Concentration and Signal Intensity in T1-Weighted MR Images. Iranian Journal of Radiology, 11, Article ID: e12667. http://dx.doi.org/10.5812/iranjradiol.12667 
[9] Sakuma, H., O’Sullivan, M., Lucas, J., Wendland, M.F., Saeed, M., Dulce, M.C., et al. (1994) Effect of Magnetic Susceptibility Contrast Medium on Myocardial Signal Intensity with Fast Gradient-Recalled Echo and Spin-Echo MR Imaging: Initial Experience in Humans. Radiology, 190, 161-166. http://dx.doi.org/10.1148/radiology.190.1.8259398

[10] Lanczi, L.I., Balázs, E., Beresova, M., Tircsó, G. and Berényi, E.L. (1970) Comparing Low-Field and High Field Relaxometry Properties of Solutions and Clinically Used Contrast Agents. European Congress of Radiology 2013.

[11] Lindegaard, H., Vallø, J., Hørslev-Petersen, K., Junker, P. and Østergaard, M. (2001) Low Field Dedicated Magnetic Resonance Imaging in Untreated Rheumatoid Arthritis of Recent Onset. Annals of the Rheumatic Diseases, 60, 770776. http://dx.doi.org/10.1136/ard.60.8.770

[12] Pope, T., Bloem, H.L., Beltran, J., Morrison, W.B. and Wilson, D.J. (2014) Musculoskeletal Imaging. Elsevier Health Sciences, Makati City.

[13] Klein, H.-M. (2016) Clinical Low Field Strength Magnetic Resonance Imaging: A Practical Guide to Accessible MRI. Springer International Publishing, Switzerland. http://dx.doi.org/10.1007/978-3-319-16516-5

[14] Nazarpoor, M., Poureisa, M. and Daghighi, M.H. (2013) Effect of Echo Time on the Maximum Relationship between Contrast Agent Concentration and Signal Intensity Using FLAIR Sequence. Iranian Journal of Medical Physics, 10, 59-67.

[15] Bjørnerud, A. (2008) The Physics of Magnetic Resonance Imaging. Department of Physics, University of Oslo, Oslo.

[16] Poh, C.K., Hardy, P.A., Liao, Z., Clark, W.R. and Gao, D. (2003) Nonintrusive Characterization of Fluid Transport Phenomena in Hollow-Fiber Membrane Modules Using MRI: An Innovative Experimental Approach. Membrane Science and Technology, 8, 89-122. http://dx.doi.org/10.1016/S0927-5193(03)80008-6

[17] Brix, G., Kolem, H., Nitz, W.R., Bock, M., Huppertz, A., Zech, C.J., et al. (2008) Basics of Magnetic Resonance Imaging and Magnetic Resonance Spectroscopy. In: Reiser, M., Semmler, W. and Hricak, H., Eds., Magnetic Resonance Tomography, Springer-Verlag, Berlin, 92-108.

[18] Jung, B.A. and Weigel, M. (2013) Spin Echo Magnetic Resonance Imaging. Journal of Magnetic Resonance Imaging, 37, 805-817. http://dx.doi.org/10.1002/jmri.24068

[19] Estelrich, J., Sánchez-Martín, M.J. and Busquets, M.A. (2015) Nanoparticles in Magnetic Resonance Imaging: From Simple to Dual Contrast Agents. International Journal of Nanomedicine, 10, 1727-1741.

[20] Giers, M.B., McLaren, A.C., Plasencia, J.D., Frakes, D., McLemore, R. and Caplan, M.R. (2013) Spatiotemporal Quantification of Local Drug Delivery Using MRI. Computational and Mathematical Methods in Medicine, 2013, Article ID: 149608. http://dx.doi.org/10.1155/2013/149608

[21] Chen, X., Astary, G.W., Sepulveda, H., Mareci, T.H. and Sarntinoranont, M. (2008) Quantitative Assessment of Macromolecular Concentration during Direct Infusion into an Agarose Hydrogel Phantom Using Contrast-Enhanced MRI. Magnetic Resonance Imaging, 26, 1433-1441. http://dx.doi.org/10.1016/j.mri.2008.04.011

[22] Li, S.K., Jeong, E.K. and Hastings, M.S. (2004) Magnetic Resonance Imaging Study of Current and Ion Delivery into the Eye during Transscleral and Transcorneal Iontophoresis. Investigative Ophthalmology \& Visual Science, 45, $1224-$ 1231. http://dx.doi.org/10.1167/iovs.03-0821

[23] Osuga, T. and Han, S. (2004) Proton Magnetic Resonance Imaging of Diffusion of High- and Low-Molecular-Weight Contrast Agents in Opaque Porous Media Saturated with Water. Magnetic Resonance Imaging, 22, 1039-1042. http://dx.doi.org/10.1016/j.mri.2003.07.004

[24] Buckley, D. and Parker, G.J. (2005) Measuring Contrast agent Concentration in T1-Weighted Dynamic Contrast-Enhanced MRI. In: Jackson, A., Buckley, D.L. and Parker, G.J.M., Eds., Dynamic Contrast-Enhanced Magnetic Resonance Imaging in Oncology, Springer, Berlin, 69-79. http://dx.doi.org/10.1007/3-540-26420-5_5

[25] Hathout, G. and Jamshidi, N. (2012) Parameter Optimization for Quantitative Signal-Concentration Mapping Using Spoiled Gradient Echo MRI. Radiology Research and Practice, 2012, Article ID: 815729. http://dx.doi.org/10.1155/2012/815729

[26] Sasaki, M., Shibata, E., Kanbara, Y. and Ehara, S. (2005) Enhancement Effects and Relaxivities of Gadolinium-DTPA at 1.5 versus 3 Tesla: A Phantom Study. Magnetic Resonance in Medical Sciences, 4, 145-149. http://dx.doi.org/10.2463/mrms.4.145

[27] Korb, J.P. and Bryant, R.G. (2002) Magnetic Field Dependence of Proton Spin-Lattice Relaxation Times. Magnetic Resonance in Medicine, 48, 21-26. http://dx.doi.org/10.1002/mrm.10185

[28] Reeder, S.B., Smith, M.R. and Hernando, D. (2016) Mathematical Optimization of Contrast Concentration for $\mathrm{t}_{1}$-Weighted Spoiled Gradient Echo Imaging. Magnetic Resonance in Medicine, 75, 1556-1564. http://dx.doi.org/10.1002/mrm.25744

[29] Brasch, R.C., Weinmann, H.J. and Wesbey, G.E. (1984) Contrast-Enhanced NMR Imaging: Animal Studies Using Gadolinium-DTPA Complex. American Journal of Roentgenology, 142, 625-630. http://dx.doi.org/10.2214/ajr.142.3.625 


\section{Submit or recommend next manuscript to SCIRP and we will provide best service for you:}

Accepting pre-submission inquiries through Email, Facebook, LinkedIn, Twitter, etc.

A wide selection of journals (inclusive of 9 subjects, more than 200 journals)

Providing 24-hour high-quality service

User-friendly online submission system

Fair and swift peer-review system

Efficient typesetting and proofreading procedure

Display of the result of downloads and visits, as well as the number of cited articles

Maximum dissemination of your research work

Submit your manuscript at: http://papersubmission.scirp.org/ 